\title{
EFFICACY OF THREE DIFFERENT RETREATMENT FILE SYSTEMS FOR GUTTA-PERCHA REMOVAL USING CONE BEAM COMPUTED TOMOGRAPHY
}

\author{
Ahmed M. Aly' ${ }^{1 *}$ BDS, Amr Mohamed Abdallah² PhD, Rania M. El Backly ${ }^{3}$ PhD.
}

\begin{abstract}
INTRODUCTION: The maximum ability of retreatment files to remove defective obturation materials is very important during endodontic retreatment.

AIM OF THE STUDY: The purpose of this study was to compare the efficiency of three different retreatment systems in root canal filling removal using chloroform as solvent.

MATERIALS AND METHODS: Thirty six extracted single rooted mandibular human premolars were used in this study following appropriate institutional ethical consent. Teeth were accessed and prepared chemo-mechanically to a standard working length of $17 \mathrm{~mm}$ using Revo-S files. Obturation was done with lateral condensation using Total Fill bioceramic sealer and gutta percha. Teeth were randomly divided into three groups $(\mathrm{n}=12)$ according to retreatment files used: ProTaper-D group, R-ENDO group and M-Two-R group using Chloroform as solvent. Specimens were labeled then preoperative and postoperative CBCT scans were conducted and volume of remaining obturation material was measured by Osirix 32-bits software to be compared. Data were collected and statistical analysis was done where $\mathrm{P}$ value was set as $\mathrm{P}<0.05$.

RESULTS: All systems significantly removed gutta percha. However, none of the used files completely removed the root canal filling material from the canals. The Protaper D group left significantly more root canal filling material than the M-Two R and R-Endo groups (P $<0.05$ ). No statistically significant difference was found between the M-Two R and R-Endo groups (Wilcoxon test used, P $<0.05$ ). ProTaper D left more remnants in the coronal third (18.48\%) showing statistically significant difference with the other groups. No Statistically significant differences were found at the middle or apical thirds between all groups (Friedman, Kruskal-Wallis tests used, $\mathrm{P}<0.05$ ).
\end{abstract}

CONCLUSION: The R-Endo and M-two R were more effective in removing root canal filling material than the ProTaper D.

KEY WORDS: Retreatment, Mtwo-R, R-Endo, Protaper-D, Bioceramic sealer.

RUNNING TITLE: Efficacy of different retreatment files in endodontic retreatment.

1 Dentist, Ministry of health, Alexandria, Egypt.

2 Professor of Endodontics, Conservative Dentistry Department, Faculty of Dentistry, Alexandria University, Alexandria, Egypt.

3 Assistant professor of Endodontics, Conservative Dentistry Department, Faculty of Dentistry, Alexandria University, Alexandria, Egypt.

*Corresponding author:

E-mail: Ahmedsaleh85@hotmail.com

\section{INTRODUCTION}

Non-surgical endodontic retreatment aims to re-establish healthy periapical tissues after poor treatment or reinfection of an obturated root canal system resulting from coronal or apical leakage (1-4). To achieve this aim, it is essential to remove the infected obturation material and dentin as well as negotiate the apical foramen to ensure patency followed by three dimensional obturation to seal the root canal system apically, coronally and even laterally (5).

Gutta percha can be removed from the root canals using hand files with heat or chemicals as solvents, but it is a time consuming and exhausting procedure (6-7). Thus, various types of motor derived retreatment files were developed to remove gutta percha from the root canals, e.g. ProTaper-D (Dentsply Maillefer, Ballaigues, Switzerland), M-Two Retreatment files (VDW, Munich, Germany) and R-Endo (Micro-Mega, Besancon, France), which could provide a faster and more convenient retreatment procedure for both clinician and patient (8).
The Protaper-D system consists of three files: D1 file has a tip diameter of 30 and taper $9 \%$ used in the coronal third, D2 file has a tip diameter of 25 and taper $8 \%$ that reachs the end of middle third and D3 file has a tip diameter of 20 and taper 7\% that reaches the full working length. The R-Endo system consists of three files: R1 file has a tip diameter of 25 and taper $8 \%$ used in the coronal third, R2 file have a tip diameter of 25 and taper $6 \%$ used at the middle third and R3 file have a tip of 25 and taper 4\% used to the full working length. The M-Two R system consists of two instruments (25/0.05 and 15/0.05) with cutting tips for efficient removal of gutta-percha fillings. The file R15/.05 is used first followed by the R25/.05 file and both files reach the full working length.

Computed tomography (CT) is an accurate non invasive imaging modality that has been used to asses the amount of root canal filling material remnants following retreatment which does not require destruction of the teeth that may alter the final results $(10,11,12)$. 
Therefore, the objective of the current study was to evaluate and compare the effectiveness of Protaper D, M-Two R and R-Endo retreatment systems for the removal of root canal filling materials during retreatment when bioceramic sealer is used.

The null hypothesis of this study is that the use of different rotary retreatment systems in the presence of chloroform will have comparable difference in the efficiency to remove the remaining filling material during retreatment procedures.

\section{MATERIALS AND METHODS}

This study was approved by the institutional ethics committee of Faculty of Dentistry, Alexandria University (International No. IORG0008839) dated on 25/10/2017. Also, informed consents were obtained before taking the samples.

The study was conducted at the Faculty of Dentistry, Alexandria, Egypt following approval of the institutional review board.

\section{Sample preparation and obturation}

Thirty six extracted single rooted human mandibular premolar teeth were used in this study collected from the Oral Surgery Department, Faculty of Dentistry, Alexandria University.

All teeth were digitally radiographed from the buccolingual and mesiodistal views using Heliodent-Plus sensor and x-ray device (Dentsply Sirona, Germany) to exclude presence of more than one root canal, calcification or resorption. Teeth were thoroughly cleaned using a hand scaler and stored in distilled water until use.

The teeth were accessed using a high speed round bur size 4 and Endo-Z bur (Dentsply Maillefer, Ballaigues, Switzerland) under water cooling.

A diamond disc was used to reduce all the teeth coronally to adjust working length to $17 \mathrm{~mm}$. After introducing manual files (\#10 and \#15) to establish a glide path, teeth were prepared chemo-mechanically using Revo-S files (MicroMega, Besancon, France) starting with the SC1 file for coronal flare followed by SC2 and SU reaching the full working length. The files were activated by a Marathon electric motor (Saeyang Microtech, Korea) at a speed of $280 \mathrm{rpm}$ and $200 \mathrm{~g} / \mathrm{cm}$ torque.

The canals were irrigated during the preparation and between each file by $2 \mathrm{ml} 5.25 \% \mathrm{NaOCl}$ (Clorox, The Egyptian Co. for household detergents, Egypt) using the luer lock syringes of 31 gauge (DiaDent Group International, Burnaby, BC, Canada).

After cleaning and shaping, all teeth were irrigated with $17 \%$ EDTA solution (Prevest Denpro limited, India) for 1 minute followed by a final rinse by $5.25 \% \mathrm{NaOCl}$ and canals were dried by paper points.

Obturation was done with lateral condensation technique using Total Fill bioceramic sealer (FKG Dentaire SA, Switzerland) and Revo-S gutta percha (MicroMega, France) no.25, taper (0.06).

Digital x-rays were taken for all samples mesiodistally and buccolingually to verify the root filling quality. All specimens were stored at $100 \%$ humidity and $37^{\circ} \mathrm{C}$ in a humidified incubator for 10 hours to allow sealer to completely set.
Three dimensional volumetric analysis of filling material using cone beam computed tomography

The specimens were fixed onto a rubber base (Zetaplus, Zhermack, Italy) blocks (5 in each block) (12) and placed on the chin rest of the J.Morita R100 (Morita 3DX; J.Morita Mfg corp., Kyoto, Japan) cone beam 3D imaging system for image attainment. Axial, frontal and sagittal sections were obtained. The scans were done with a field of view (FOV) of $100 \mathrm{~mm} \times \mathrm{H}$ $80 \mathrm{~mm}$. The volumes of interest were then reconstructed with $0.260 \mathrm{~mm}$ isometric voxel size. The tube voltage was $90 \mathrm{kVp}$ and $8 \mathrm{~mA}$ and the exposure time was 9.4 seconds.

Primary CBCT images were used to evaluate the volume of root canal filling using Osirix 32 bits software (Pixmeo SARL, Geneva, Switzerland) (Fig.1.A)

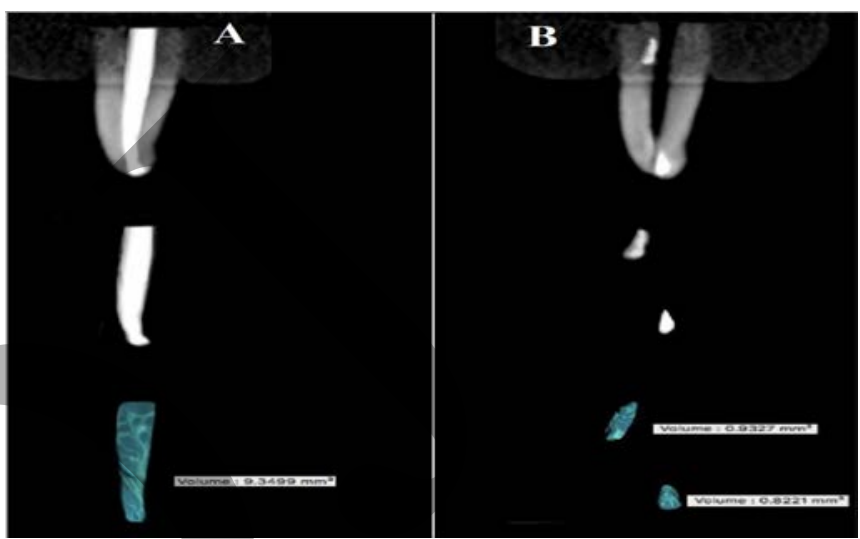

Figure (1): Images for a representative sample from the REndo group obtained by C.B.C.T. and Osirix software to evaluate root canal filling volume. (A) before retreatment; (B) after retreatment.

\section{Removal of obturation materials}

Samples were randomly divided into three groups $(n=12)$ according to the files used in the retreatment procedure:

ProTaper D group (Dentsply Maillefer, Ballaigues, Switzerland), R-Endo group (Micro-Mega, Besancon, France) and M-Two R group (VDW, Munich, Germany).

Two to three drops of chloroform (Al-Gomhoria chemical co. ltd, Egypt) were introduced into the canals and left to act for 1 minute before removal of root canal filling material. Each file was then introduced for three pecks before it was removed and wiped with gauze.

Retreatment was done with the following sequence: Protaper-D group: The D1 file was first used till it reached the coronal third of the canal, D2 file progressed to the middle third followed by the D3 file which then reached the full length of the canals. R-Endo Group: The R1 instrument was used in the coronal third followed by the R2 file that progressed to the middle third, the R3 file was used to the full working length. M-Two Retreatment Group: The file R15/.05 was used first followed by the R25/.05 file where both reached the full working length.

For all groups, irrigation was done using $2 \mathrm{ml}$ of $5.25 \%$ $\mathrm{NaOCl}$ during preparation and between each file using luer lock endodontic syringe and needle (gauge 31 by DiaDent Group International, Burnaby, BC, Canada). Retreatment procedure for all groups was considered finished when the working length was reached and no more filling material was observed on the files. 
After retreatment another CBCT scan of each root was taken with the same position of the initial CBCT scanning.

The volume of filling material inside the canal was measured again after retreatment using the Osirix 32 bits software (Fig.1.B). The volume percentage of the remaining root canal filling was calculated using the following equation:

Volume of the remaining root canal filling

Volume of the original root canal filling

\section{Statistical Analysis}

Sample size calculation was done using G-Power 3.1.9.2 software (Universität Düsseldorf, Germany).

After Data were collected and coded, they were subjected to statistical analysis using the SPSS IBM version 20 software. Graphs were then constructed using Microsoft Excel software (Microsoft Corporation, New Mexico, USA)/ SPSS software (IBM Corporation, NYC, USA).

All statistical analysis was done using two tailed tests with an alpha error of 0.05 . Descriptive statistics in the form of mean with standard deviation and median for scale data was done. Median was the measure of concern.

To test for differences of median between study groups and the measured outcome, the Kruskal-Wallis test was used while the Wilcoxon test was used to test for changes of median volume before and after intervention. The Friedman test was used for all groups to test for differences of volume percentage of filling material remaining between the coronal, middle and apical zones per group while the Kruskal-Wallis test was used to compare between groups at each zone.

\section{RESULTS}

Regarding the amount of filling material identified in the canals before and after retreatment, it was found that all groups significantly removed the filling material when comparing between pre and post-retreatment CBCT images (Table 1) $(\mathrm{P}<0.05)$.

Table (1): Describes the (Mean, SD and Median) of the volume in $\mathrm{mm} 3$ of the filling material in each group before and after retreatment

\begin{tabular}{|c|c|c|c|c|c|}
\hline \multicolumn{2}{|c|}{ Volume } & M-Two R & ProTaper D & R-Endo & ${ }^{\mathrm{H}} \mathbf{P}$ \\
\hline \multirow{3}{*}{ Before } & $\begin{array}{l}\text { Min.- } \\
\text { Max. }\end{array}$ & $3.092-12.400$ & $3.658-12.300$ & $6.763-13.000$ & \multirow{3}{*}{$\begin{array}{c}4.062 \\
(0.131)\end{array}$} \\
\hline & $\begin{array}{l}\text { Mean } \\
\pm \text { S.D }\end{array}$ & $8.364 \pm 2.933$ & $7.269 \pm 2.433$ & $9.194 \pm 1.960$ & \\
\hline & Median & 8.645 & 7.082 & 8.925 & \\
\hline \multirow{3}{*}{ After } & $\begin{array}{l}\text { Min.- } \\
\text { Max }\end{array}$ & $0.350-3.394$ & $0.454-2.900$ & $0.119-2.102$ & \multirow{3}{*}{$\begin{array}{c}6.698 \\
(0.035)^{*}\end{array}$} \\
\hline & $\begin{array}{l}\text { Mean } \\
\pm \text { S.D }\end{array}$ & $1.028 \pm 0.859$ & $1.632 \pm 0.784$ & $0.931 \pm 0.646$ & \\
\hline & Median & 0.720 & 1.641 & 1.012 & \\
\hline \multicolumn{2}{|l|}{$\mathrm{Z}$ (P) } & $3.059(0.002) *$ & $3.059(0.002)^{*}$ & $3.059(0.002) *$ & \\
\hline
\end{tabular}

There was a statistically significant difference between the volumes of the remaining filling materials between the Protaper D group and the two other groups. More remaining filling material remained following the use of the ProTaper D system (23.91\%) followed by less filling material using the M-Two R system (12.53\%) and the R-Endo system (11.19\%) showing no statistically significant difference between them (Table 2).

The ProTaper Universal retreatment system left more remnants in the coronal third (18.48\%) which showed statistically significant difference with the two other groups. Less coronal remnants were found in the M-Two R group (4.483\%) and the R-Endo group (3.40\%) with no statistically significant difference between them. (Table 3)

In the middle third there was no statistically significant difference between the groups. However, M-Two R left more remnants $(4.75 \%)$, followed by the ProTaper Universal retreatment system (2.64\%). While the R-Endo left the least remnants (1.64\%). (Table 3)

Regarding the apical third, there was no statistically significant difference between any of the groups, yet the R-Endo system left more remnants (6.15\%), followed by the M-Two R (3.21\%) and finally the ProTaper universal system left the least remnants (2.64\%). (Table 3)

Table (2): Describes the (Mean, SD, and Median) of the volume by $\%$ of the remaining filling material in each group.

\begin{tabular}{|l|c|c|c|c|}
\hline Volume \% & M-Two R & ProTaper D & R-Endo & HP \\
\hline $\begin{array}{l}\text { Min.- } \\
\text { Max. }\end{array}$ & $4.28-27.37$ & $7.61-38.64$ & $1.09-31.08$ & \\
\cline { 1 - 4 } $\begin{array}{l}\text { Mean } \pm \\
\text { S.D }\end{array}$ & $12.532 \pm 7.492$ & $23.91 \pm 11.450$ & $11.19 \pm 8.801$ & \multirow{2}{*}{$7.641(0.022)^{*}$} \\
\cline { 1 - 4 } Median & 11.395 & 25.22 & 11.525 & \\
\hline
\end{tabular}

Z: Wilcoxon test

H: Kruskal-Wallis test

$* \mathrm{P}<0.05$ (significant)

Table (3): Represents the volume \% of remaining filling material in the three groups regarding coronal, middle and apical zones.

\begin{tabular}{|c|c|c|c|c|c|}
\hline \multicolumn{2}{|c|}{ Volume } & M-Two R & ProTaper D & R-Endo & ${ }^{\mathrm{H}} \mathbf{P}$ \\
\hline \multirow{2}{*}{ Coronal } & $\begin{array}{l}\text { Min.- } \\
\text { Max. }\end{array}$ & $0-11.47$ & $0-38.64$ & $0-22.03$ & \multirow{2}{*}{$\begin{array}{r}15.108 \\
(0.001)^{*}\end{array}$} \\
\hline & $\begin{array}{r}\text { Mean } \pm \\
\text { S.D }\end{array}$ & $4.483 \pm 4.555$ & $18.484 \pm 11.932$ & $3.398 \pm 6.517$ & \\
\hline \multirow{2}{*}{ Middle } & $\begin{array}{l}\text { Min.- } \\
\text { Max. }\end{array}$ & $0-23.53$ & $0-22.06$ & $0-17.10$ & \multirow{2}{*}{$\begin{array}{r}0.392 \\
(0.822)\end{array}$} \\
\hline & $\begin{array}{r}\text { Mean } \pm \\
\text { S.D }\end{array}$ & $4.839 \pm 9.182$ & $2.783 \pm 6.890$ & $1.644 \pm 4.895$ & \\
\hline \multirow{2}{*}{ Apical } & $\begin{array}{l}\text { Min.- } \\
\text { Max. }\end{array}$ & $0-15.17$ & 0-20.99 & $0-14.47$ & \multirow{2}{*}{$\begin{array}{r}5.578 \\
(0.061)\end{array}$} \\
\hline & $\begin{array}{r}\text { Mean } \pm \\
\text { S.D }\end{array}$ & $3.210 \pm 5.272$ & $2.642 \pm 5.995$ & $6.153 \pm 5.345$ & \\
\hline \multicolumn{2}{|l|}{$X^{2}(\mathbf{P})$} & $1.077(0.584)$ & $13.512(0.001)^{*}$ & $7.800(0.020) *$ & \\
\hline
\end{tabular}

X2: Friedman test

$\mathrm{H}$ : Kruskal-Wallis test

$\mathrm{P}<0.05$ (significant) 


\section{DISCUSSION}

Retreatment of cases present with previous endodontic treatment failure continues to pose a challenge to daily endodontic practice. The success of non-surgical root canal retreatment depends on the complete removal of root canal filling material that harbors necrotic tissues and bacteria resulting in apical inflammation. Also, to allow negotiation of the apical foramen, efficient cleaning, shaping, and filling of the root canal system (2-13).

The current study aimed to compare three different rotary file systems designed for retreatment which are the ProTaper D,( ProTaper D, www.Dentsplysirona.com) R-Endo (R-Endo, www.micro-mega.com )and M-Two R (M-Two R, www.vdw-dental.com). Manufacturers of the mentioned systems claimed the improved efficiency in removing root canal filling during retreatment procedures.

Upon comparing pre and post-retreatment CBCT images; we found that the volume of obturation material before and after retreatment significantly decreased, showing that all groups significantly removed the filling material during retreatment procedures.

Also, the ProTaper-D group showed significantly higher volume of remaining obturation material compared with the other groups mainly found in the coronal third of the tested samples. Thus, the null hypothesis of the present study was rejected.

Various systems have been applied for the removal of root canal filling material from canals which includes stainless steel hand files, Ni-Ti files, ultrasonic files, and lasers. Usage of conventional files has been found to be a quite tedious and time-consuming procedure for the operator to remove a well-condensed obturating material and might also cause endodontic mishaps. Authors like Giuliani et al (14), Somma F et al (2008) (15), Reddy N et al (2013) (16), Neelam Mittal et al (2014) (17) and Mercy Joseph et al (2016) (18) found that rotary file systems were more effective in removing root canal filling material than hand files. The current study results correspond to these findings stating that rotary files can be efficient in removing root canal filling material.

$\mathrm{Ni}-\mathrm{Ti}$ files possess super-elasticity which allows more centered canal preparations with less transportation of the canal and a decreased incidence of canal aberrations. Furthermore, the increased taper preparation facilitates adequate irrigation. These files have a greater cutting efficiency once engaged in a crown-down approach and a continuous reaming motion. Consequently, rounder root canal preparations, with less straightening and a smaller amount of apical extrusion, are achievable. In spite of their increased flexibility, separation is still a concern with $\mathrm{Ni}-\mathrm{Ti}$ files (19).

To increase safety during retreatment procedures and to produce more adequate shaping, retreatment files have been designed with non-cutting tips, radial lands, varying tapers and rake angles, and changing pitch lengths (13).

In the present study Revo-S files were used in the initial canal preparation of the sample teeth. Revo-S system was developed with a distinctive asymmetric cross-section intended to decrease the stress on the instrument. Revo-S is designed with less taper files and thus less dentin removal and less tapered canal preparation than other systems such as
ProTaper and i-RaCe at all the different thirds (20).

In the present study single rooted human mandibular premolars with single canals were used as it is a common choice for extraction in orthodontic treatment and thus can be obtained in a sound condition (21).

In addition, root canals with oval and long oval shaped cross sections such as those of the mandibular premolars are complex and challenging to treat or retreat, even for the most competent endodontic practitioners and even in spite of the ever expanding endodontic instruments. They often present with buccal and lingual surface extensions, which may be difficult, if not impossible to access (22).

Samples were decoronated with a diamond disk to standardize the working length $(17 \mathrm{~mm})$ to minimize variations by eliminating some variables, such as crown anatomy and root length, thus providing a reliable comparison of the tested retreatment systems $(23,24)$.

Different methods have been used to evaluate the cleaning of the root canals after retreatment procedures. The most common methods have been used were the longitudinal sectioning of the roots $(25,26)$, radiographic assessment $(27,28)$, teeth clearing technique (1) and computed tomography scan (29).

Each technique has its limitations, Schirrmeister et al., 2006 reported that residual material might be lost by splitting the roots longitudinally. Also, recent studies have shown that the use of conventional two-dimensional (2D) radiographs failed to evaluate the actual amount of remaining obturation material within the canals and thus the effectiveness of retreatment procedures $(30,27)$.

Based on that, in the present study C.B.C.T. imaging was used to evaluate the remaining filling material allowing to view samples in three dimensions (3D) without any intervention that may alter results later on. This has been previously demonstrated by Gad et al in 2016(12).

The present study compared the differences in volume of remaining obturation material before and after retreatment procedure and showed that all groups significantly removed the filling material when comparing between pre and postretreatment CBCT images $(\mathrm{P}<0.05)$. Both systems, R-Endo and $\mathrm{M}$-Two $\mathrm{R}$ left the least remnants of obturation material with no statistically significant difference.

The R-endo system showed the least mean area of remaining gutta-percha; this may be due to its design that has different tapers and same tip diameter of $0.25 \mathrm{~mm}$ increasing the rigidity especially in the $4 \%$ taper files (31).

It seems that some design features of M-Two retreatment files in comparison to ProTaper $\mathrm{R}$ result in better performance of these files in removing obturation material. Mtwo $\mathrm{R}$ files have positive rake angle with two cutting edges, an increasing pitch length in the apical-coronal direction and S-shaped cross-section. As they have sharp blades, it is capable to cut through obturation material and reach the apical terminal by gliding through the canal (9, 32). Also, unlike the ProTaper R instruments, Mtwo R files do not require a crown-down instrumentation sequence (9).

However, the ProTaper retreatment group left the highest volume of obturating material remnants and from the results of the present study we can conclude that the design of retreatment files could have an effect on its cleaning ability. The increased taper of the ProTaper R files (compared with less taper design of the M-Two $\mathrm{R}$ and R-Endo files) would 
subsequently increase the thickness of its core resulting in reduced flexibility of the files. Thus, the brushing motion in retreatment of mandibular premolars with high prevalence to have oval canals would be less effective especially at the coronal third which may justify the results of this study.

Similar results of the Protaper retreatment files regarding the high volume of the remaining obturating material were shown in Hulsmann et al and Shaheen et al studies (3, 31).

In contrast to the present study results, it was reported that retreatment with ProTaper files showed no difference with $\mathrm{H}$ files, Race group or Flex Master group concerning cleanliness (1).

This may be due to the fact that those studies performed further preparations following the retreatment process $(15,31)$. However, it is also probable that the reason could be the difference in the type of canals tested in the other studies; mesiobuccal canals of mandibular molars in contrast to oval canals of mandibular premolars used in the current study (12). Some authors also concluded that a hybrid instrumentation technique with rotary instruments followed by finishing with hand instruments may improve the cleaning procedure to obtain the maximum results (33).

The Gad et al study results were in contrast with the present study results as it showed no statistically significant difference between the Protaper D universal files specially designed for retreatment and the two other reciprocating systems (WaveOne and Reciproc) designed for conventional root canal treatment. Also, no solvents were used in Gad's study (12). Again, this may be due to the difference in the samples used.

Also, Gu et al study showed that the better performance of ProTaper Universal retreatment instruments may be related to their taper design. D1, D2 and D3 have three progressive tapers and lengths (33). This design may enable the instruments to remove not only GP but also the superficial layer of dentine during root filling removal (34).

Chloroform was used as a solvent in the present study. It was applied as 1-2 drops at the orifice of the samples and left to act for 1 minute to aid in softening of the GP and to facilitate the introduction of the retreatment files at the coronal access of the canal. The use of solvent in the present study may have resulted in GP and sealer adherence to the canal walls. The reason for residual GP and sealer could be attributed to the solvent ability to soften GP and modify its structure to increase the viscosity and adhesion of the obturation material, which makes it more difficult to remove especially in oval canals (35).

Few studies assessed the retreatment outcome when bioceramic sealers have been used in obturation. The studies concluded that there is no significant difference between different files used in retreatment regarding the volume of remaining filling material measured. However, in the present study the R-Endo and M-Two $\mathrm{R}$ files have been more efficient in retreatment than the Protaper D files (36, 37).

In this study no instruments were separated during the retreatment procedure. The low torque setting of the device improved the tactile sensation, gave better control during instrumentation and also decreased the risk of instrument separation. The motor speed was adjusted to the manufacturer's recommendation for each system. The use of each files set for only five canals and the use of the chloroform may have reduced the incidence of instrument fracture (38).

However, there were some limitations that are defined in this study such as; the inability of all the three retreatment file systems to completely remove the entire root canal filling material. This may be due to the bonding quality and strength of the bioceramic sealer used in this study.

Also, one of the limitations was the inability of the C.B.C.T. images to distinguish the difference between the gutta percha and the bioceramic sealer used.

\section{CONCLUSION}

Within the limitations of the present study, it was concluded that all of the tested systems effectively removed root canal filling during retreatment procedures. Also, it showed that the M-Two and R-Endo retreatment systems were more efficient than the ProTaper D system in removal of obturation material.

\section{Conflict of interest}

The authors declare that they have no conflicts of interest.

\section{REFERENCES}

1. Schirrmeister J, Wrbad KT, Meyer K, Altenburger M, Hellwig E. Efficacy of different rotary instruments for gutta-percha removal in root canal retreatment. J Endod. 2006;32:469-72.

2. Mollo A, Botti G, Goldoni NP, Randellini E, Paragliola $\mathrm{R}$, Chazine M, et al. Efficacy of two Ni-Ti systems and hand files for removing gutta-percha from root canals. Int Endod J. 2012;45:1-6.

3. Hulsmann M, Bluhm V. Efficacy, cleaning ability and safety of different rotary NiTi instruments in root canal retreatment. Int Endod J. 2004;37:468-76.

4. Saad AY, Al-Hadlaq SM, Nasser H, Al-Katheeri. Efficacy of two rotary NiTi instruments in the removal of gutta-percha during root canal retreatment. J Endod. 2007;33:38-41.

5. Marfisi K, Mercade M, Plotino G, Duran-Sindreu F, Bueno R, Roig M. Efficacy of three different rotary files to remove gutta percha and Resilon from root canals. Int Endod J. 2010;43:1022-8.

6. Imura N, Kato AS, Hata GI, Uemura M, Toda T, Weine F. A comparison of the relative efficacies of four hand and rotary instrumentation techniques during endodontic retreatment. Int Endod J. 2000;33:361-6.

7. Schirrmeister JF, Hermanns P, Meyer KM, Goetz F, Hellwig E. Detectability of residual Epiphany and guttapercha after root canal retreatment using a dental operating microscope and radiographs--an ex vivo study. Int Endod J. 2006;39:558-65.

8. Unal GC, Kaya B, Tac AG, Kececi AD. A comparison of efficacy of conventional and new retreatment instruments to remove gutta-percha in curved root canals, an ex vivo study. Int Endod J. 2009;42:344-50.

9. Yadav P, Bharath MJ, Sahadev CK, Makonahalli Ramachandra PK, Rao Y, Ali A, et al. An in vitro CT Comparison of Gutta-Percha Removal with Two Rotary Systems and Hedstrom Files. Iran Endod J. 2013;8:59-64.

10. Barletta FB, Rahde Nde M, Limongi O, Moura AA, Zanesco C, Mazocatto G. In vitro comparative analysis of 2 mechanical techniques for removing gutta-percha 
during retreatment. J Can Dent Assoc. 2007;73:65-9.

11. Khedmat S, Azari A, Shamshiri AR, Fadae M, Fakhar HB. Efficacy of ProTaper and Mtwo Retreatment Files in Removal of Gutta-percha and GuttaFlow from Root Canals. Iran Endod J. 2016;11:184-9.

12. Gad HM, Zaazou AM, El Backly RM. Reciprocation versus rotary motion for endodontic retreatment. Alex Dental J. 2016;41:72-7.

13. Saad AY, Al-Hadlaq SM, Al-Katheeri NH. Efficacy of two rotary NiTi instruments in the removal of gutta-percha during root canal retreatment. J Endod. 2007;33:38-41.

14. Giuliani V, Cocchetti R, Pagavino G. Efficacy of ProTaper universal retreatment files in removing filling materials during root canal retreatment. J Endod. 2008;34:1381-4.

15. Somma F, Cammarota G, Plotino G, Grande NM, Pameijer $\mathrm{CH}$. The effectiveness of manual and mechanical instrumentation for the retreatment of three different root canal filling materials. J Endod. 2008;34:466-9.

16. Reddy N, Admala SR, Dinapadu S, Pasari S, Reddy MP, Rao MS. Comparative analysis of efficacy and cleaning ability of hand and rotary devices for gutta-percha removal in root canal retreatment: an in vitro study. J Contemp Dent Pract. 2013;14:635-43.

17. Mittal N, Jain J. Spiral computed tomography assessment of the efficacy of different rotary versus hand retreatment system. J Conserv Dent. 2014;7:8-12.

18. Joseph M, Ahlawat J, Malhotra A, Rao M, Sharma A, Talwar S. In vitro evaluation of efficacy of different rotary instrument systems for gutta percha removal during root canal retreatment. JCED. 2016;8:e355-e60.

19. Bergmans L, Van Cleynenbreugel J, Wevers M, Lambrechts P. Mechanical root canal preparation with NiTi rotary instruments: rationale, performance and safety. Status report for the American Journal of Dentistry. Am J Dent. 2001;14:324-33.

20. Deka A, Bhuyan AC, Bhuyan D. A comparative evaluation of root canal area increase using three different nickel-titanium rotary systems: An ex vivocone-beam computed tomographic analysis. Contemp Clin Dent. 2015;6:79-83.

21. Zachrisson BU. Clinical outcome with mandibular second versus first premolar extractions in orthodontic treatment. World J Orthod. 2005;6:296-303.

22. Jensen LE. Mechanical preparation of oval-shaped root canalsin mandibular premolars with the TRUShape 3DConforming File: a micro-computed tomography study. University of Iwoa. USA. 2017.

23. Tasdemir T, Kursat Er, Celik D, Yildirim T. Effect of passive ultrasonic irrigation on apical extrusion of irrigating solution. Eur J Dent. 2008;2:198-203.

24. Al-Sudani D. Radiovisiography versus conventional radiography for estimation of canal length: an in vitro study. Pakistan Oral \& Dent J. 2002;22:171-73.

25. Kosti E, Lambrianidis T, Economides N, Neofitou C. Ex vivo study of the efficacy of $\mathrm{H}$-files and rotary $\mathrm{Ni}$ Tiinstruments to remove gutta-percha and four types of sealer. Int Endod J. 2006;39:48-54.

Alexandria Dental Journal. Volume 45 Issue 3.
26. Zmener O, Pameijer CH, BanegasG. Retreatmentefficacy of hand versus automated instrumentation in oval-shaped root canals: An ex vivo study. Int Endod J. 2006;39:521-6.

27. De Carvalho Maciel AC, Zaccaro Scelza MF. Efficacy of automated versus hand instrumentation during root canal retreatment: An ex vivo study. Int Endod J. 2006;39:779-84.

28. Masiero AV, Barletta FB. Effectiveness of different techniques for removing gutta-percha during retreatment. Int Endod J. 2005;38:2-7.

29. Barletta FB, Rahde Nde M, Limongi O, Moura AA, Zanesco C, Mazocatto G. In vitro comparative analysis of 2 mechanical techniques for removing gutta-percha during retreatment. J Can Dent Assoc. 2007;73:65.

30. Kfir A, Tsesis I, Yakirevich E, Matalon S, Abramovitz I. The efficacy of five re-treatment techniques: Microscopic vs. radiographic evaluation. Int Endod J. 2012;45:35-41.

31. Ali SM, Pradeep PS, Paul S, Jhons DA, Ganesh PB. Comparative evaluation of efficiency and time of gutta percha removal using hedstorm files, protaper retreatment and r-endo files-an ex vivo study. JEBMH. 2015;49:8484-7.

32. Marques da Silva B, Baratto-Filho F, Leonardi DP, Henrique Borges A, Volpato L, Branco Barletta F. Effectiveness of ProTaper, D-RaCe, and Mtwo retreatment files with and without supplementary instruments in the removal of root canal filling material. Int Endod J. 2012;45:927-32.

33. Gu LS, Ling JQ, Wei X, Huang XY. Efficacy of ProTaper Universal rotary retreatment system for GP removal from root canals. Int Endod J. 2008;41:288-95.

34. Malhotra K, Taneja S, Kumar P. Comparison of efficacy of hand and rotary instrumentation for removing a resinbased obturating material (EndoRez) in curved root canals during retreatment: An in vitro study. Endodontol. 2017;29:60-4.

35. Bhagavaldas MC, Diwan A, Kusumvalli S, Pasha S, Devale M, Chava DC. Efficacy of two rotary retreatment systems in removing Gutta-percha and sealer during endodontic retreatment with or without solvent: A comparative in vitro study. J Conserv Dent, 2017;20:12-6.

36. Aksel H, Küçükkaya Eren S, Askerbeyli Örs S, Serper A, Ocak M, Çelik HH. Micro-CT evaluation of the removal of root fillings using the ProTaper Universal Retreatment system supplemented by the XP-Endo Finisher file. Int Endod J. 2019;52:1070-6.

37. Romeiro K, de Almeida A, Cassimiro M, Gominho L, Dantas E, Chagas N, et al. Reciproc and Reciproc Blue in the removal of bioceramic and resin-based sealers in retreatment procedures. Clin Oral Investig. 2019;1-12.

38. Yared GM, Bou Dagher FE, Machtou P. Failure of Profile instruments used with high and low torque motors. Int Endod J. 2001;34:471-5. 